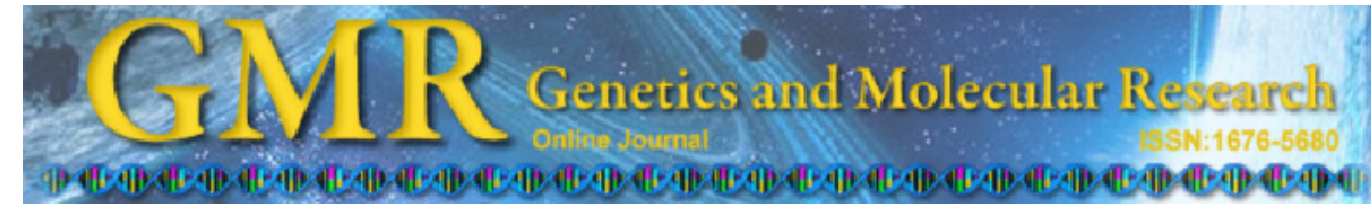

Methodology

\title{
An improved strategy based on RAPD markers efficiently identified 95 peach cultivars
}

\author{
M.L. Yu' ${ }^{1}$, W.Y. Wang ${ }^{2,3}$, R.J. Ma ${ }^{1}$, Z.J. Shen ${ }^{1}$ and J.G. Fang ${ }^{2,3}$ \\ ${ }^{1}$ Institute of Horticulture, Jiangsu Academy of Agricultural Sciences, \\ Nanjing, P.R. China \\ ${ }^{2}$ College of Horticulture, Nanjing Agricultural University, Nanjing, P.R. China \\ ${ }^{3}$ Jiangsu Fruit Crop Genetics Improvement and Seeding Propagation \\ Engineering Center, Nanjing, China \\ Corresponding author: M.L. Yu \\ E-mail: mly1008@yahoo.com.cn
}

Genet. Mol. Res. 11 (2): 1158-1168 (2012)

Received May 9, 2011

Accepted December 15, 2011

Published May 7, 2012

DOI http://dx.doi.org/10.4238/2012.May.7.1

\begin{abstract}
DNA markers have useful applications in cultivar identification. A novel analysis approach called cultivar identification diagram (CID) was developed using DNA markers in the separation of plant individuals. This new strategy is less time- and cost-consuming, has reliable results, and was constructed for fingerprinting. Ten 11-mer primers were used to amplify the genotypes; all 95 peach genotypes (from the National Peach Germplasm Repository, in Nanjing, China) were distinguished by a combination of 54 primers. The utilization of the CID among these 95 peach cultivars was also verified by the identification of three randomly chosen groups of cultivars. This identification showed some advantages including the use of fewer primers and easy separation of all cultivars by the corresponding primers marked in the right position on the CID. This peach CID could provide the information to separate any peach cultivars of these 95, which may be of help to the peach industry in China and for the utilization of DNA markers to identify other plant species.
\end{abstract}

Key words: Peach; RAPD; Cultivar identification; Molecular markers 


\section{INTRODUCTION}

Peach (Prunus persica (L.) Batsch) belongs to the subfamily Prunoideae in the Rosaceae family. Together with other species, including plums, cherries, and apricots, they are referred to as "stone fruits". Peaches were probably the first fruit crop domesticated in China about 4000 years ago (Bunyard, 1938). As the center for the origin of peaches, China has the most abundant resources of both wild and cultivated varieties (Yuan et al., 2002; Yang et al., 2002). Unlike cultivars of other tree fruits in the same family that are mostly the result of chance selection, many peach cultivars were bred. Peaches are a self-pollinated species with a high degree of self-compatibility and homozygosity (Baird et al., 1996). In addition, most of the existing peach varieties (cultivars) were bred from very limited parental material, which resulted in a narrow genetic base for peach cultivars. This extensively decreased the genetic diversity among peach cultivars. The low degree of heterogeneity makes it very difficult to differentiate genotypes. Accurate identification of varieties and genotypes is therefore essential for patent protection of these materials. Traditionally, identification of fruit trees including peach was based on morphological or physiological aspects. Biochemical markers like isoenzymes have been used but they have many drawbacks like the limited number of polymorphisms detected between close cultivars and variations due to the physiological stage. Even though several generations of DNA markers have been developed and used for cultivar identification and genetic analysis leading to publication of thousands of papers, no efficient approach has been developed or used for cultivar identification apart from the phylogenetic trees and/or some fingerprints employed on a very small scale. Phylogenetic trees cannot tell us which primer to use or the information to use or refer to for specific cultivar identification, while traditional fingerprinting does not present all the fingerprints together and is thus not ideal for identification of many cultivars.

Various DNA-based markers have recently been developed and used for studies on genetic diversity, fingerprinting and cultivar origins (D'Onofrio et al., 2009; Melgarejo et al., 2009; Cheng and Huang, 2009; Elidemir and Uzun, 2009; Papp et al., 2010). Randomly amplified polymorphic DNA (RAPD) markers used in peach have proven to be a reliable marker system for genetic fingerprinting and also in determining the genetic relationships among germplasm collections. RAPD markers have the advantage of being simple, able to detect relatively small amounts of genetic variation and do not need prior information on the genome. However, RAPDs do not give information about the genome. The technique has already been successfully applied to estimate genetic relationships in apricot (Mariniello et al., 2002), mulberry (Vijayan, 2004), grape (Benjak et al., 2005), figs (Sadder and Ateyyeh, 2006), apple (StarkUrnau, 2002a), litchi (Ding et al., 2000), cherry (Demirsoy et al., 2008), Indian cashew (Archak et al., 2003), longan (Yonemoto et al., 2006), olive (Belaj et al., 2003), and pear (Stark-Urnau, $2002 \mathrm{~b}$; Lee et al., 2004). The stable and reproducible marker systems can be used for proper identification in peach varieties (Byrne, 1990; Yamamoto et al., 2005). In China, most of the molecular marker analyses of peaches have been performed using RAPD (Yang et al., 2001; Sun et al., 2005; Zong et al., 2005). Only a few studies have attempted to analyze genetic diversity, and determine genetic relationships and genetic mapping of peach germplasm resources. DNA fingerprinting is a powerful tool currently applied widely in forensic science that can also be equally useful in the genotyping of plants. Among the many markers available, RAPD (Williams et al., 1990) markers are useful for cultivar analysis with great advantages in simplicity, efficiency, relative ease of execution and no need for any previous sequence information. Optimization of the RAPD technique by choosing $11 \mathrm{nt}$ primers and strict screening PCR an- 
nealing temperature before it is employed in fingerprinting plants can make RAPD a preferable technique for use in plant cultivar identification. In practice, the powerful DNA markers available for plant identification have not made fruit crop variety identification an efficient, recordable, and as easy as a task desired, a really awkward situation facing fruit and other crop scientists owing to the potential of this technique in commercial production. The main reason for this situation is the dearth of analysis strategies for DNA fingerprints. The currently popular analysis techniques for DNA banding patterns known as cluster analyses cannot be applied efficiently in cultivar or species separation. This has made the utilization of DNA markers in crop and seed identification unpopular in practice.

China is an important agricultural country in the world and has abundant plant resources, which makes distinguishing plant cultivars or varieties an important task for plant scientists. One of the ultimate purposes of plant science is to serve agriculture and the practical application of new biological techniques for agricultural production is very important. Since the development of DNA markers, they have been applied in plant cultivar identification, which is thought to be one of the main uses of DNA markers. However, the scale of this application of DNA markers in cultivar identification of plant and related work is limited by the tedious, time-consuming, and non-repeatable nature of the techniques. There was therefore a need to address these drawbacks and make DNA marker techniques workable and as easy as measuring something using a ruler. Here, we introduce a much more workable method that can be readily used in plant cultivar identification on a scale like the scheme shown in Figure 1. This method makes the identification of peach cultivars a practical, efficient, recordable, and easily referable task. A cultivar identification diagram (CID) was generated and can be utilized as source of reference information for peach cultivar identification in the future. The diagram shows the separation of 95 selected peach cultivars based on RAPD banding patterns and can definitely be of great service to the peach industry worldwide.

\section{MATERIAL AND METHODS}

\section{Plant materials}

Young leaves of 95 selected peach cultivars were collected from the Peach National Germplasm Nursery, Jiangsu Academy of Agricultural Science, Nanjing, China. The names and origins of these cultivars are shown in Table 1.

\section{Genomic DNA extraction}

Total genomic DNA of each genotype was extracted from young leaves using the modified cetyltrimethylammonium bromide (CTAB) method (Murray and Thompson, 1980; Bousquet et al., 1990). The extracted DNA was diluted to a final concentration of $30 \mathrm{ng} / \mu \mathrm{L}$ with $1 \mathrm{X}$ TE buffer and stored at $-20^{\circ} \mathrm{C}$ pending use.

\section{RAPD analysis}

In case of RAPD reactions, 54 primers were tested with a few genotypes initially and only those primers resulting in clear unambiguous banding patterns with all genotypes tested 
were selected for use with the full set of genotypes.

Fifty-four 11-mer RAPD primers were used for screening in this study. In order to increase credibility of the fragments, we used only those primers resulting in clear unambiguous banding patterns. Subsequently, 10 primers (Table 2) that showed well-resolved and reproducible bands were selected to assay all genotypes, while the others were discarded. Reaction solutions consisted of $2.0 \mu \mathrm{L} 10 \mathrm{X}$ buffer, $1.2 \mu \mathrm{L} 25 \mathrm{mM} \mathrm{MgCl}_{2}, 1.6 \mu \mathrm{L} 2.5 \mathrm{mM}$ dNTP, $1.6 \mu \mathrm{L}$ $1.0 \mu \mathrm{M}$ primer, $0.1 \mu \mathrm{L} 5 \mathrm{U} / \mu \mathrm{L}$ rTaq Polymerase Dynazyme and $1 \mu \mathrm{L}$ genomic DNA, making a total volume of $20 \mu \mathrm{L}$. Amplification reactions were performed based on the standard protocol of Williams et al. (1990), with minor modifications. PCR was carried out in an Autorisierter Thermocycler (Eppendorf, Hamburg, Germany), programmed as follows: an initial pre-denaturation step for $5 \mathrm{~min}$ at $94^{\circ} \mathrm{C}$; then, 42 cycles each consisting of a denaturation step for $30 \mathrm{~s}$, an annealing step for $1 \mathrm{~min}$ at annealing temperature and an extension step for $2 \mathrm{~min}$ at $72^{\circ} \mathrm{C}$ (Table 2). Amplification was terminated by a final extension at $72^{\circ} \mathrm{C}$ for $10 \mathrm{~min}$. After amplification, the amplified DNA fragments were separated by gel electrophoresis on $1.3 \%$ agarose $(\mathrm{w} / \mathrm{v})$ (Figure 1) in 1X TAE buffer (0.04 M Tris-acetate, 0.001 M EDTA, pH 8.0) at $100 \mathrm{~V}$. The gels were stained with $0.5 \mu \mathrm{g} / \mathrm{mL}$ ethidium bromide and visualized under ultraviolet light for polymorphic bands among the cultivars. In order to have reproducible, accurate and clear banding patterns, all amplifications were repeated separately at least thrice.

\section{Data analysis}

Only clear unambiguous bands in the photographic prints of gels were chosen and scored for cultivar identification. Where some cultivars had a specific band in the fingerprint generated from one primer, they could be separated singly, and those cultivars sharing the same banding pattern were separated into the same sub-group. Based on this strategy, all the peach cultivars were gradually and completely separated from one another as more primers were employed.

\section{Test of utilization and workability of the CID}

Three groups of peach cultivars, randomly chosen from the inter- and intra-groups, were used to verify the utilization and workability of the diagram showing the separation of the 95 cultivars. The three groups of cultivars were marked " $\mathrm{A}$ ", "B" and "C" and the corresponding primers to be used for separation of each group were easily picked out from the diagram. If the randomly chosen cultivars could be distinguished accurately and quickly as anticipated on the whole cultivar identification diagram, it would definitely provide assurance that the strategy developed and employed in this study is scientific, workable and efficient, consequently making this method an ideal way to use molecular markers for identification of fruit crop cultivars and seed samples of field crops. The data from the cultivar separation in the cultivar identification diagram can also be generated into a database for ease of reference.

\section{RESULTS}

\section{Cultivar identification}

To establish a stable and optimistic RAPD system with high reproducibility, longer 
primers (11 nt) were employed and the annealing temperatures for each primer were screened based on the quality and reproducibility of banding patterns. The primers were randomly screened from a stock of 54 11-mer primers, and once an optimistic primer that could produce reproducible polymorphic bands was screened, it was further utilized in the identification of the peach cultivars. By the time the 11th primer was screened and utilized, all the 95 peach cultivars had been successfully identified. An example of the RAPD patterns in the study includes that obtained using primer Y41 (Figure 1), which was also the first primer used to amplify the 95 peach cultivars. The electrophoresis results show that primer Y41 generated uniform, clear, and reproducible band patterns in 33 peach cultivars assigned with lane number codes 2, 3, 9, $11,18,19,28,31,32,36,38,40,41,42,44,45,47,49,50,51,53,59,63,68,70,75,77,81$, $82,83,91,93$, and 95 (the lane numbers correspond to the cultivar names as shown in Table 1). This group of 33 peach cultivars was easily differentiated from the other 62 cultivars by the presence or absence of a distinct 2000-bp band, causing all the 95 cultivars to be separated into two groups. The other 9 primers (Table 2) were, step by step, screened and chosen to differentiate the peach cultivars until full separation was achieved. The second primer (Y23) used, for example, could further separate the two groups of cultivars earlier identified by primer Y41 into smaller groups. Other primers were chosen to differentiate the various subgroups of peach cultivars. The group of three cultivars ('Mei guo hong pan tao', 'Bai tao' and 'Da hong hua'), could be separated with a band size of about 850 and 950 bp (Figure 2A) by primer Y42 while the other group of 'Shu guang' and 'Jin tong 6 hao' could be separated with a band of about $1500 \mathrm{bp}$ (Figure 2B) by primer Y29. After these, the peach cultivars could still be separated into many more groups and following this method systematically and utilizing 10 primers, all the 95 peach cultivars could eventually be differentiated from each other (Figure 3).

$\begin{array}{llllllllllllllllllllllllll}\mathrm{M} & 1 & 2 & 3 & 4 & 5 & 6 & 7 & 8 & 9 & 10 & 11 & 12 & 13 & 14 & 15 & 16 & 17 & 18 & 19 & 20 & 21 & 22 & 23 & 24\end{array}$

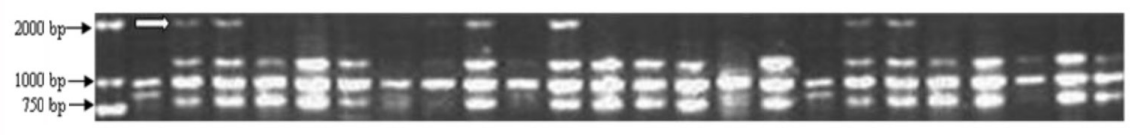

M 252627282930313233343536373839404142434445464748

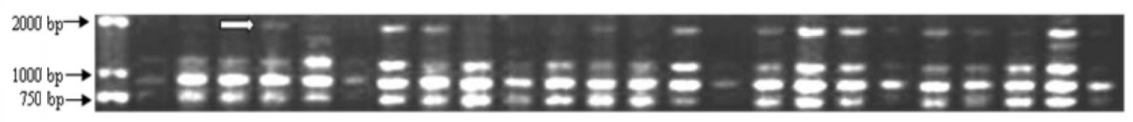

M 495051525354555657585960616263646566676869707172

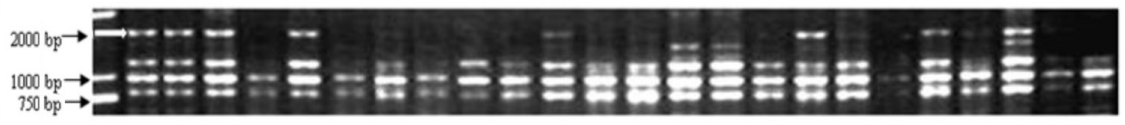

M 7374757677787980818283848586878889909192939495

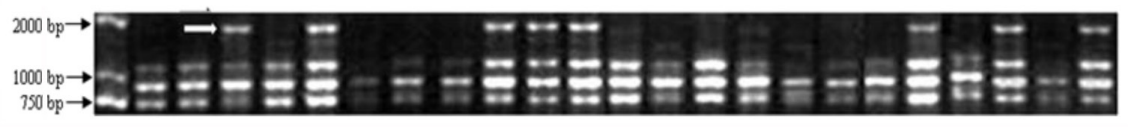

Figure 1. DNA banding patterns of 95 fruiting peach cultivars amplified by primer Y41. Lane $M=$ DL2000 plus DNA ladders; lanes 1-95 = accession numbers of fruiting peach cultivars listed in Table 1, same as those in the following figures. 
Table 1. Name of the materials used in this experiment.

\begin{tabular}{|c|c|c|c|}
\hline No. & Cultivar & No. & Cultivar \\
\hline 1 & "Mei guo hong pan tao" & 49 & "Jin tong 5 hao" \\
\hline 2 & "Xia guang" & 50 & "Jin tong 6 hao" \\
\hline 3 & "Jing chun" & 51 & "Zao yi nv" \\
\hline 4 & "Feng hua pan tao" & 52 & "Da hong hua" \\
\hline 5 & "Rui guang mei yu" & 53 & "Liang ji" \\
\hline 6 & "Bei nong 1 hao" & 54 & "Feng huang shui mi" \\
\hline 7 & "Zhao hui" & 55 & "Bai hua" \\
\hline 8 & "Mei gui hong" & 56 & "Fei cheng bai li" \\
\hline 9 & "Xia hui 6 hao" & 57 & "Feng hua yu lu (early)" \\
\hline 10 & "Xia hui 1 hao" & 58 & "Zao hua lu" \\
\hline 11 & "Jin luo man" & 59 & "Chun hua" \\
\hline 12 & "Zao xia lu" & 60 & "Zao mei" \\
\hline 13 & "Mei xiang" & 61 & "Feng hua yu lu (late)" \\
\hline 14 & "Chun lei” & 62 & "Shen zhou bai mi" \\
\hline 15 & "Wan bai hua" & 63 & "Rui guang 19 hao" \\
\hline 16 & "Yuan dong bai tao" & 64 & "Xia hui 5 hao" \\
\hline 17 & "Hua yu lu" & 65 & "Chun xue" \\
\hline 18 & "Fei cheng 39 hao" & 66 & "Bu mu zao sheng" \\
\hline 19 & "Chun xia mi" & 67 & "Xiang jiao tao" \\
\hline 20 & "Xia hui 2 hao" & 68 & "Xin da jiu bao" \\
\hline 21 & "Jing yu" & 69 & "Zhao xia" \\
\hline 22 & "Lai shan mi" & 70 & "Jin shan zao hong" \\
\hline 23 & "Zao hong lu" & 71 & "You ming bai tao" \\
\hline 24 & "Feng bai" & 72 & "Sha zi zao sheng" \\
\hline 25 & "Jing mi" & 73 & "Bai mang pan tao" \\
\hline 26 & "Hu you 003" & 74 & "Tang xing bai hua" \\
\hline 27 & "Yin hua lu" & 75 & "Yu hua 2 hao" \\
\hline 28 & "Yu hua lu" & 76 & "Ying qing" \\
\hline 29 & "Shen zhou hong mi" & 77 & "Zao hong 2 hao" \\
\hline 30 & "Da jiu bao" & 78 & "Nong shen" \\
\hline 31 & "Da zhen ban chi yue" & 79 & "Bai feng 2 hao" \\
\hline 32 & "Xiao" & 80 & "Xia hui 7 hao" \\
\hline 33 & "Qian dai ji" & 81 & "Xiao hong hua" \\
\hline 34 & "Cang fang zao sheng" & 82 & "Fu lei de li ke" \\
\hline 35 & "Ri chuan bai feng" & 83 & "Run zhou shui mi" \\
\hline 36 & "A bu bai tao" & 84 & "Hong gan lu" \\
\hline 37 & "Bai tao" & 85 & "Xin bai hua" \\
\hline 38 & "Jing yan" & 86 & "Jing hong" \\
\hline 39 & "Zi jin hong 2 hao" & 87 & "Yu hua 3 hao" \\
\hline 40 & "Rui guang 12 hao" & 88 & "Bei nong 2 hao" \\
\hline 41 & "Ri ben bai feng" & 89 & "Fei cheng 47 hao" \\
\hline 42 & "Hu jing mi lu" & 90 & "Jin hua lu" \\
\hline 43 & "Yang shan 2 hao" & 91 & "Du bai feng" \\
\hline 44 & "Da tuan mi lu" & 92 & "Chun yan" \\
\hline 45 & "Gang shan zao sheng" & 93 & "Zao feng wang" \\
\hline 46 & "Gang shan bai" & 94 & "An nong shui mi" \\
\hline 47 & "Shu guang" & 95 & "Shuang xi hong" \\
\hline 48 & "Zi jin hong 1 hao" & & \\
\hline
\end{tabular}

Table 2. Ten primers were chosen for further fingerprinting of the 95 peach genotypes.

\begin{tabular}{llr}
\hline Primer & Nucleotide sequence $\left(5^{\prime}-3^{\prime}\right)$ & Annealing temperature $\left({ }^{\circ} \mathrm{C}\right)$ \\
\hline Y-3 & GTTTCGCTCCA & 44.7 \\
Y-5 & GTTTCGCTCCG & 43.7 \\
Y-17 & AGGGGTCTTGG & 41.7 \\
Y-18 & AGGGGTCTTGC & 41.7 \\
Y-22 & GGACCCAACCT & 42.8 \\
Y-23 & GGACCCAACCG & 43.7 \\
Y-29 & GTGTGCCCCAG & 42.8 \\
Y-34 & AAGCCTCGTCT & 44.4 \\
Y-41 & AGCGTCCTCCG & 43.7 \\
Y-42 & AGCGTCCTCCC & 44.8 \\
\hline
\end{tabular}



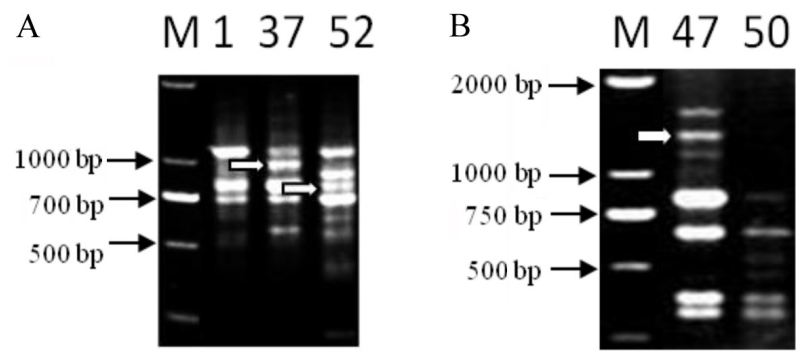

Figure 2. DNA banding patterns of 3 fruiting peach cultivars in group A from the separation of primer Y42 and 2 fruiting peach cultivars in group B separated by Y29. Lane $M=$ DL2000 plus DNA ladders. The bands pointed to with larger arrows were those employed to separate the cultivars. For lanes identification, see Table 1.

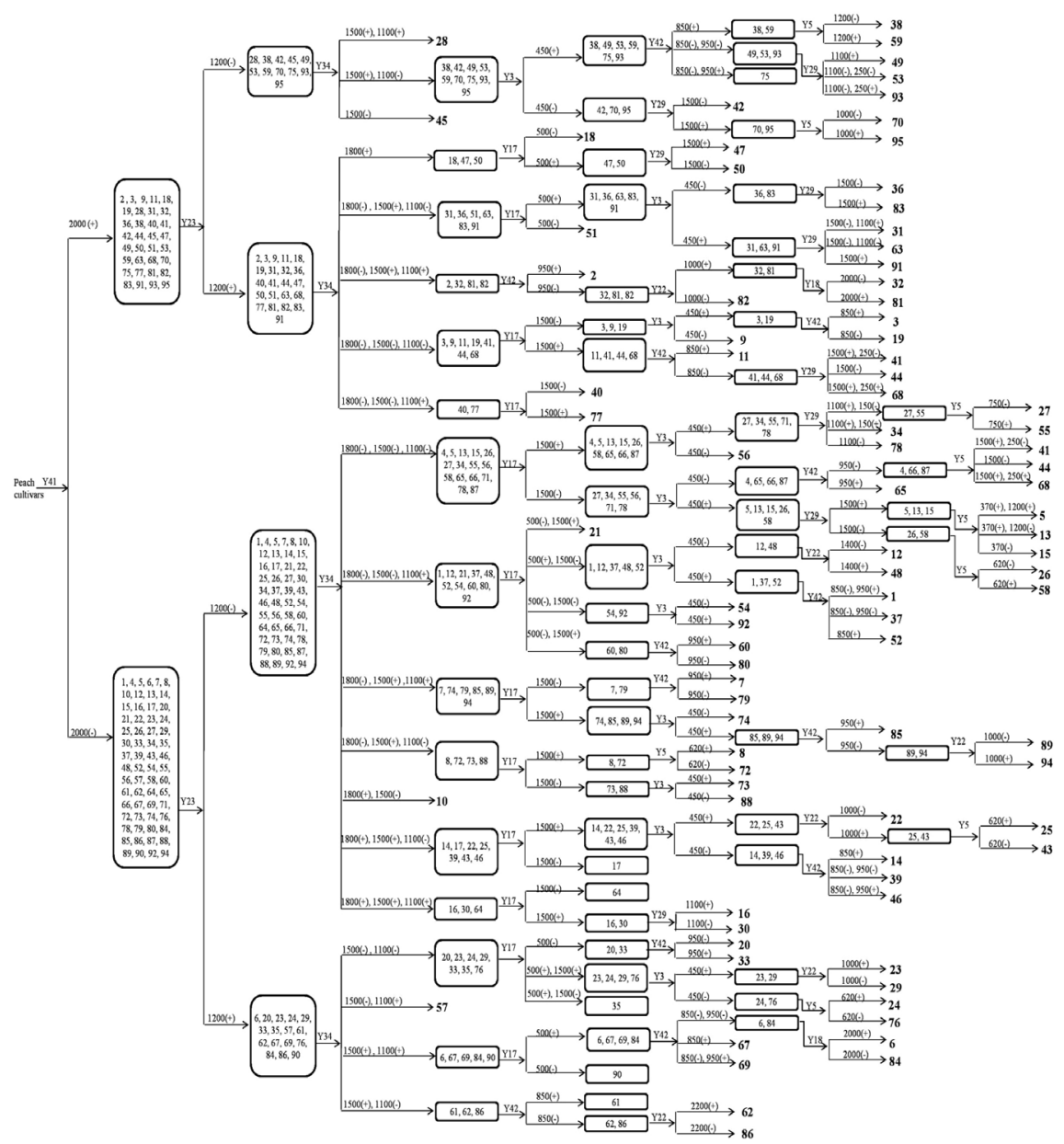

Figure 3. Cultivar identification diagram of the fruiting peach cultivars by 10 primers. The numbers above each horizontal line in the diagram were the size of the polymorphic bands used to separate the cultivars following the line, and the unit of them was in bp. (+) means presence of the polymorphic band; (-) means absence of it. The cultivar numbers in bold are those that were separated. 


\section{Test of the utilization and workability of the CID}

The important aim of this study was not just how to use RAPD markers to distinguish the 95 peach cultivars as done by most earlier reports focusing on the utilization of DNA markers in identifying plant cultivars, but to also generate a referable peach CID test that could be used in the practical identification of some of these cultivars in the future for the nursery industry as well as in cultivar-right-protection. With these two aims in mind, it was critical to verify the utilization, workability and efficiency of the CID test. To undertake this, three groups of cultivars comprised of 'Mei guo hong pan tao', 'Xia guang', 'Feng hua pan tao', and 'Jing chun'; 'Mei gui hong' and 'Sha zi zao sheng'; 'Xiao' and 'Xiao hong hua', which came from the inter- and intra-groups in the CID, were randomly chosen and used for the verification exercise. From the location of these cultivars in CID, it was easy to find the primer to use in separating them. Primers Y41, Y34, Y17, Y5, and Y18 were used, as projected, to separate the three groups of cultivars. Clearly, the verified PCR results agree with those anticipated in the CID in that all cultivars in these three groups were separated accordingly. The first group according to Figure 4A, B and C can be validated with three primers including Y41, Y34 and Y17. The PCR results showed that four peach cultivars could first be separated into two groups by primer Y41 with a band of about $2000 \mathrm{bp}$. One group made of 'Xia guang' and 'Feng hua pan tao' can be further disjoined with primer Y34 with a 1100-bp band. The other group of 'Mei guo hong pan tao' and 'Jing chun' could then be divided by use of primer Y17 with a band of 1500 bp. 'Mei gui hong' and 'Sha zi zao sheng' could be separated with a specific band of about 620 bp of primer Y5 as in the CID. Another group of 'Xiao' and 'Xiao hong hua' was divided by primer Y18 with the band of about $2000 \mathrm{bp}$. By this procedure, all three groups were successfully identified with the suitable combination of primers. This test therefore proves the availability, workability and efficiency of this method in peach cultivar identification.
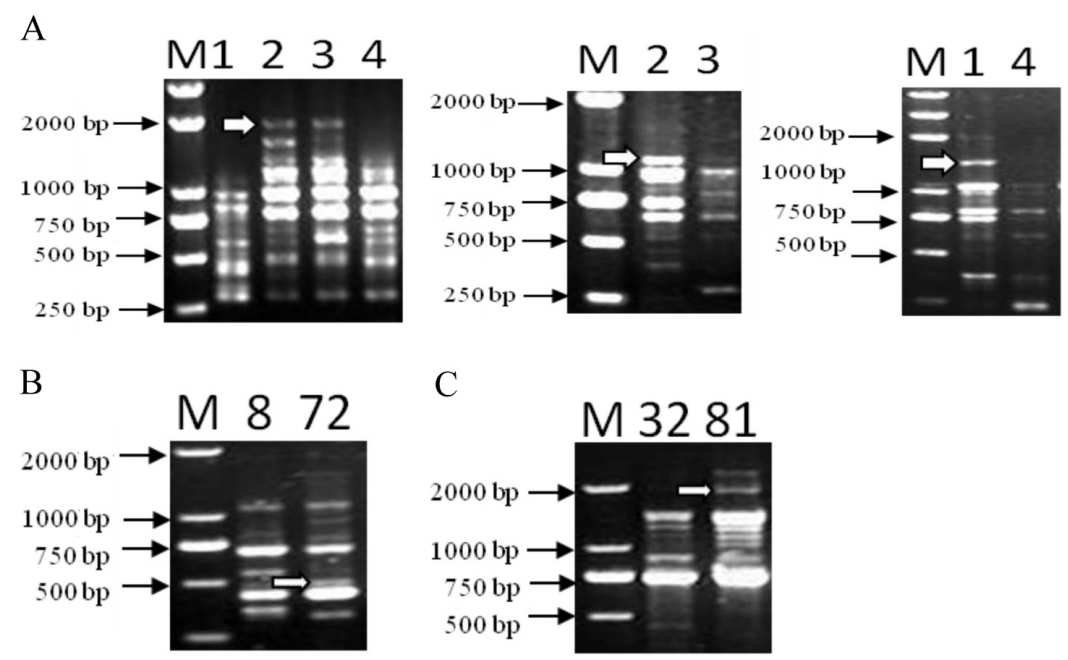

$\mathrm{C}$

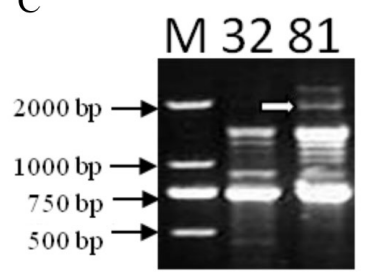

Figure 4. Verification result of two cultivars selected randomly by the corresponding primers. Lane $M=$ DL2000 plus marker. A. Fingerprint obtained with the three primers used to separate the first group of cultivars selected. "a", "b", and "c" were obtained with primers Y41, Y34, and Y17, respectively. B. Fingerprint obtained with the primer (Y5) used to separate the group "Mei gui hong" and "Sha zi zao sheng". C. DNA fingerprint obtained with the primer (Y18) used to separate the group "Xiao" and "Xiao hong hua". For lanes identification, see Table 1. 


\section{DISCUSSION}

Peach is one of the oldest fruit trees with a 4000-year cultivation history where it has been subjected to long-term natural selection and production practices that evolved around the characteristics of different groups and species. A frequent exchange of resources between regions coupled with a lack of management of varietal resources make background knowledge unclear. The name confusion, especially in nursery stock markets is a real problem as a myriad false claims of inferiority or superiority of a cultivar are not backed by any evidence. This can adversely affect future production of desired cultivars. The need to demystify science by developing strategies for applying new knowledge and technology to practical activities to solve the above problem is therefore both necessary and significant. DNA markers are a powerful technique that can be used to accurately identify plant cultivars and species, with several generations of DNA markers having been developed and used for cultivar identification (Saker et al., 2006; Chiu et al., 2010) and genetic analysis (Boronnikova et al., 2007; Silvestrini et al., 2008; Bhau et al., 2009; Baysal et al., 2010). Thousands of papers on this subject have also been published. Nonetheless, DNA markers have not been readily used for genotyping of plants. In fact, the situation is much more dire than anticipated, with the question of whether DNA markers can be adequately and easily used in the identification of plant varieties yielding a negative response. No efficient approach has been developed to employ DNA markers easily and efficiently in plant cultivar identification except where phylogenetic tree clusters or some fingerprints were utilized. Apparently, clusters formed in phylogenetic trees cannot tell us which information can be used for identification of the desired plant samples while fingerprinting cannot present all the fingerprints from many cultivars together for identification. The main reasons for these weaknesses can be attributed to the fact that no analysis could connect the information of DNA fingerprints with cultivars in an easy, clear and readable way. The new approach we have developed to utilize DNA marker in distinguishing cultivars is both efficient and practical, as well as less expensive, faster and clearer, among other benefits. This strategy can realize the power of DNA markers on plant cultivar identification, and can use the polymorphic nature of each primer, gradually distinguishing and charting every species. Although the method does not accurately reflect the genetic relationship of the cultivars/species, theoretically, the first cultivars/species to be separated will have the widest genetic distance between them. The reverse of this holds true. This method is definitely a great help in plant cultivar identification for purposes of cultivar-right-protection, and early identification of seedlings in the nursery industry. To improve the efficiency and utilization of this strategy in the fruit crop industry, more commercially important cultivars in production were chosen for identification. If there are one or more new cultivars that need to be separated and the identification result put in the diagram, the whole set of the primers used to run the additional DNA samples can be used and a larger diagram with the identification information of the new cultivars formed. If the set of primers used originally, such as the 10 in this study, do not work well in the separation of new cultivars, new primers need to be screened and used. This research is not difficult or time-consuming and deserves a place in agriculture.

This is the first report on using RAPD primers in sequence to identify peach cultivars. In order to verify the reliability of this theory, experimental verification is very necessary. In this study, the experimental verification returned satisfactory results. Therefore, this experiment confirms the possibility of utilizing DNA markers even in plant species, which have a 
highly heterozygous genome, without requiring a genetic linkage map and/or any DNA sequence information to distinguish the cultivars. It appears to be an effective technique for convenient development of selection markers in fruit trees. This approach has been tested and proven to be a more efficient way of distinguishing plant cultivars with referable information. It is not just a simple theoretical model but a truly handy one as it makes DNA markers more applicable for plant variety identification in practice. At present, we have initiated similar research to present all the identification information of most fruit crop cultivars cultivated in China for cultivar-right-protection and better service for the nursery and general fruit industry. Furthermore, the polymorphic bands generated may be developed into special molecular markers for identification of the cultivars in the future. We believe that as research progresses, we can use this method to draw a table for each species, whether plant, animal or any other creature, which can work just like a periodic table in providing the information needed to separate the species/cultivars as desired.

In conclusion, this method is rapid, simple and produces reliable results, since it was possible to demonstrate that a standard set of primers can be used to distinguish many Prunus persica L. species.

\section{ACKNOWLEDGMENTS}

Research supported by the National Natural Science Foundation of China (\#30871681) and the China Agriculture Research System (\#CARS-31).

\section{REFERENCES}

Archak S, Gaikwad AB, Gautam D, Rao EVVB, et al. (2003). DNA fingerprinting of Indian cashew (Anacardium occidentale L.) varieties using RAPD and ISSR techniques. Euphytica 130: 397-404.

Baird WV, Ballard RE, Rajapkse S and Abbott AG (1996). Progress in Prunus mapping and application of molecular markers to germplasm improvement. Hortic. Sci. 31: 1099-1106.

Baysal O, Siragusa M, Gumrukcu E, Zengin S, et al. (2010). Molecular characterization of Fusarium oxysporum $\mathrm{f}$. melongenae by ISSR and RAPD markers on eggplant. Biochem. Genet. 48: 524-537.

Belaj A, Satovic Z, Ismaili H, Panajoti D, et al. (2003). RAPD genetic diversity of Albanian olive germplasm and its relationships with other Mediterranean countries. Euphytic 130: 387-395.

Benjak A, Ercisli S, Vokurka A, Maletic E, et al. (2005). Genetic relationships amonggrapevine cultivars native to Croatia, Greece and Turkey. Vitis 44: 73-77.

Bhau BS, Medhi K, Das Ambrish P, Saikia SP, et al. (2009). Analysis of genetic diversity of Persea bombycina "Som" using RAPD-based molecular markers. Biochem. Genet. 47: 486-497.

Boronnikova SV, Kokaeva ZG, Gostimsky SA, Dribnokhodova OP, et al. (2007). Analysis of DNA polymorphism in a relict Uralian species, large-flowered foxglove (Digitalis grandiflora Mill.), using RAPD and ISSR markers. Russ. J. Genet. 43: 530-535.

Bousquet J, Simon L and Lalonde M (1990). DNA amplification from vegetative and sexual tissues of tree using polymerase chain reaction. Can. J. For. Res. 20: 254-257.

Bunyard EA (1938). The history and cultivation of the peach and nectarine. J. Royal Hort. Sci. 63: 114-121.

Byrne DH (1990). Isozyme variability in four diploid stone fruits compared with other woody perennial plants. J. Hered. 81: 68-71.

Cheng ZP and Huang HW (2009). SSR fingerprinting Chinese peach cultivars and landraces (Prunus persica) and analysis of their genetic relationships. Sci. Hortic. 120: 188-193.

Chiu T, Pang J, Chen M and Tsen H (2010). Improvement of strain discrimination by combination of RAPD with PFGE for the analysis of the swine isolates of Salmonella enterica serovar Choleraesuis. World J. Microbiol. Biotechnol. 27: 465-469.

D’Onofrio C, Lorenzis G, de Giordani T, Natali L, et al. (2009). Retrotransposon-based molecular markers in grapevine 
species and cultivars identification and phylogenetic analysis. Acta Hortic. 827: 45-52.

Demirsoy L, Demir T, Demirsoy H, Kacar YA, et al. (2008). Identification of some sweet cherry cultivars grown in Amasya by RAPD markers. Acta Hortic. 795: 147-152.

Ding XD, Lu LX, Chen XJ and Guan X (2000). Identifying litchi cultivars and evaluating their genetic relationships by RAPD markers. J. Trop. Subtrop. Bot. 8: 49-54.

Elidemir AY and Uzun I (2009). Assessment of genetic diversity of some important grape cultivars, rootstocks, and wild grapes in Turkey using RAPD markers. Acta Hortic. 827: 275-278.

Lee GP, Lee CH and Kim CS (2004). Molecular markers derived from RAPD, SCAR, and the conserved 18S rDNA sequences for classification and identification in Pyrus pyrifolia and P. communis. Theor. Appl. Genet. 108: 14871491.

Mariniello L, Sommella MG, Sorrentino A, Forlani M, et al. (2002). Identification of Prunus armeniaca cultivars by RAPD and SCAR markers. Biotechnol. Lett. 24: 749-755.

Melgarejo P, Martcnez JJ, Hernández Fca, Martínez R, et al. (2009). Cultivar identification using 18S-28S rDNA intergenic spacer-RFLP in pomegranate (Punica granatum L.). Sci. Hortic. 120: 500-503.

Murray MG and Thompson WF (1980). Rapid isolation of high molecular weight plant DNA. Nucleic Acids Res. 8: 43214325.

Papp N, Szilvassy B, Abranko L, Szabo T, et al. (2010). Main quality attributes and antioxidants in Hungarian sour cherries: identification of genotypes with enhanced functional properties. Int. J. Food Sci. Technol. 45: 395-402.

Sadder MT and Ateyyeh AF (2006). Molecular assessment of polymorphism among local Jordanian genotypes the common fig (Ficus carica L.). Sci. Hortic. 107: 347-351.

Saker MM, Adawy SS, Mohamed AA and El-Itriby HA (2006). Monitoring of cultivar identity in tissue culture-derived date palms using RAPD and AFLP analysis. Biol. Plantarum 50: 198-204.

Silvestrini M, Maluf MP, Silvarolla MB, Guerreiro-Filho O, et al. (2008). Genetic diversity of a Coffea germplasm collection assessed by RAPD markers. Genet. Res. Crop Evol. 55: 901-910.

Stark-Urnau M (2002a). Use of RAPD-markers in Malus x domestica (apple) and Pyrus communis (pear) for cultivar identification - Part I: Malus x domestica (apple). RAPD-Marker bei Malus x domestica (Apfel) und Pyrus communis (Birne) als Mittel zur Sortenidentifizierung - Teil I: Malus x domestica (Apfel). Erwerbsobstbau 44: 139-144.

Stark-Urnau M (2002b). Use of RAPD-Markers in Malus x domestica (apple) and Pyrus communis (pear) for cultivar identification - Part II: Pyrus communis (Birne). RAPD-Marker bei Malus x domestica (Apfel) und Pyrus communis (Birne) als Mittel zur Sortenidentifizierung - Teil II: Pyrus communis (Birne). Erwerbsobstbau 44: 167-171.

Sun P, Li W, Jiang HY and Yao JC (2005). Analysis of genetic relationship among cutlivars of Prunus persica using RAPD markers. J. Gansu Agric. Univ. 40: 586-590.

Vijayan K (2004). Genetic relationships of Japanese and Indian mulberry (Morus spp.) genotypes revealed by DNA fingerprinting. Plant Systemat. Evol. 243: 221-232.

Williams JG, Kubelik AR, Livak KJ, Rafalski JA, et al. (1990). DNA polymorphisms amplified by arbitrary primers are useful as genetic markers. Nucleic Acids Res. 18: 6531-6535.

Yamamoto T, Yamaguchi M and Hayashi T (2005). An intergrated genetic linkage map of peach by SSR, STS, AFLP and RAPD. J. Jpn. Soc. Hortic. Sci. 74: 204-213.

Yang XG, Zhang KC, Qin L and Wang YX (2001). RAPD analysis of germplasm resources on peach. J. Fruit Sci. 18: 276-279.

Yang YJ, Zhang KC and Lin K (2002). Studies on RAPD polymorphisms and genetic relationship of Prunus persica plants. J. Henan Agric. Univ. 36: 187-189.

Yonemoto Y, Chowdhury AK, Kato H and Macha MM (2006). Cultivars identification and their genetic relationships in Dimocarpus longan subspecies based on RAPD markers. Sci. Hortic. 109: 147-152.

Yuan Z, Luo LS, Xiao DX and Zhang DB (2002). A study on the genetic relationship of peach species using RAPD markers. Acta Agric. Univ. Jiangxiensis 24: 172-175.

Zong CW, Gao HN, Zhao CR, Wang C, et al. (2005). Studies on analysis of peach cultivars based on RAPD markers. $J$. Nanjing Agric. Univ. 28: 35-39. 